\title{
Analysis of Various Technologies for Packet Length Optimization in Wireless Sensor Network
}

\author{
Ruchira Deshmukh ${ }^{1}$, M. B. Tamboli ${ }^{2}$ \\ ${ }^{1}$ Department of Computer Engg, G.H. Raisoni Coll of Engg \& Mgt, Ahemadnagr, India \\ ${ }^{2}$ Professor, Department of Information Technology, G.H. Raisoni Coll of Engg \& Mgt, Vagholi, India
}

\begin{abstract}
In wireless sensor networks, dynamic packet length control is more efficient in terms of channel utilization and energy efficiency. In previous packet length optimizations for sensor network often employ a fixed optimal packet length scheme, while dynamic packet length provide accurate radio link estimation and increase system throughput. The adaptation of dynamic packet length is 802.11 wireless systems. The packet delivery ratio keep high i.e. $95 \%$ above and link estimated error within $10 \%$ for $95 \%$ link. The experiments provide optimization of dynamic packet length achieves best performances related to the previous experiments.
\end{abstract}

Keywords: Dynamic packet length optimization, wireless sensor network, aggregation, fragmentation, delivery ratio

\section{Introduction}

A fundamental challenge in wireless networks is that radio links are subject to transmission power, fading, and interference, which degrade the data delivery performance. This challenge is exacerbated in wireless sensor networks (WSNs), where severe energy and resource constraints preclude the use of many sophisticated techniques that may be found in other wireless systems [1][3].For example,

(i) bit rate adaptation protocols, demand special hardware that is not available on general sensor nodes;

(ii) effective forward error correction (FEC) requires the amount of redundant data transmitted to be tuned to match the link qualities which is difficult to achieve in dynamic WSNs[3].

802.11 wireless LANs were originally designed for small networks with limited traffic, and are thus not optimized for high traffic situations. However, as wireless LANs become increasingly ubiquitous, the design limitations become greatly stressed. One often neglected tunable parameter is MAC layer packet length. While packet length can be variable in the 802.11 standard, it is most often simply set to the maximum value to reduce the impact of overhead. This is indeed the optimal setting for a scenario with a single pair of nodes with a strong channel; however, in scenarios with hidden nodes and weaker channels, shorter packets may be preferable due to their lower susceptibility to loss. A hidden node for a given node $\mathrm{A}$ transmitting to a node $\mathrm{B}$ in a wireless network is one which is capable of interfering with the reception of the packet at $\mathrm{B}$, while being unable to sense the transmission of A. This ISSN 2348 - 7968 can lead to staggered collisions, which occur when node A's packet is interrupted by the hidden node, causing reception to fail. The risk of this type of loss increases with packet length because as length increases, the hidden node is required to remain silent for a longer period of time. Additionally, longer packets are more susceptible to loss due to channel errors because they require the successful decoding of more symbols[10]. Packet length adaptation seeks to address the tradeoff between lower overhead for long packet lengths, and lower probability of loss for shorter packet lengths. There has been a significant amount of research on packet length adaptation. In current packet length adaptation literature, a simple packet loss model is typically used, assuming the channel to have a constant biterror rate (BER), and neglecting staggered collisions $[1,2]$. This assumes that most packet losses occur due to random bit errors in the packet payload. However, it has experimentally been shown that for lower modulation rates in 802.11a, most packet losses occur due to failure to synchronize to the packet preamble. This type of loss cannot be accounted for using a constant BER model, as it requires a model of channel fading[2]. The purpose of this study is to find the optimal packet length for the real time channel conditions. The basic idea is: if the packet length is too large, the packet retransmission rate will be high due to packet error; on the other hand, if the packet length is too small, the effective data throughput will be low since much transmission is spent on packet headers. Therefore some optimal packet length exists to achieve maximal throughput[10]. A packet optimization scheme applicable in sensor networks must have the following important features.

(i) Dynamic packet length adaptation. Prior work in sensor networks uses fixed packet length optimization schemes. These schemes are not preferred due to the spatial temporal diversity of link qualities in WSNs.

(ii) Accurate link estimation. The performance improvement of the packet length adaptation scheme is highly dependent on the link estimation accuracy. Prior work in wireless systems does not consider unique characteristics of WSNs, e.g., resource constraints of sensor nodes, thus leading to inaccurate link estimation in sensor networks.

(iii) Easy to use. To the best of our knowledge, no prior work addresses the application programmability issues of packet length adaptation scheme in sensor networks. Without substantial programming efforts, there is still a huge gap between theoretical optimality and practically achievable gains.

The rest of the paper is organized as follows. Section II discussed related work. In section III comparative analysis of various techniques with the help of table. In section IV 


\section{International Journal of Science and Research (IJSR) \\ ISSN (Online): 2319-7064}

Index Copernicus Value (2013): 6.14 | Impact Factor (2014): 5.611

research design is explained. Finally dynamic packet optimization scheme it proposes.

\section{Related Work}

\section{A. Packet Loss Model}

Losses in Wireless LANs can be broadly classified into two types: collisions, which are the result of unfavorable traffic conditions, and channel errors, which are the result of unfavorable channel conditions. A collision occurs when a node's packet overlaps in time with that of another node which is spatially close enough to the destination to interfere. A channel error occurs when the SNR of a received packet is low due to a large path loss or a deep multipath fade. The total packet loss probability $P L$ can be computed as:

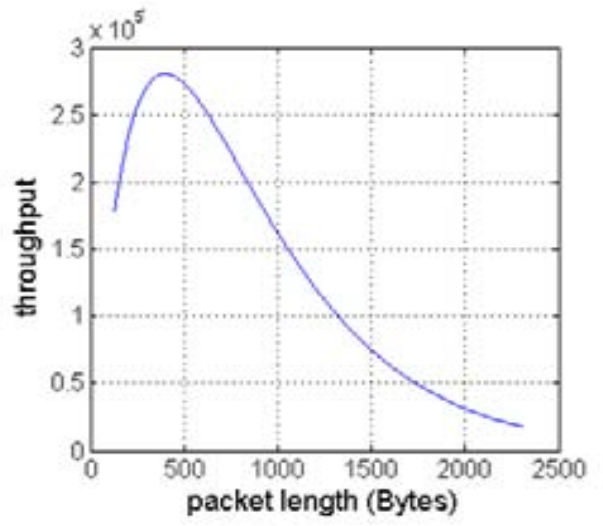

$P L=1-(1-P C)(1-P e)$

where $P C$ is the probability of collision, and $P e$ is the probability of channel error, which is assumed to be independent of $P C$.

In this analysis, we assume that all collided packets are lost, not captured, and that the probability of ACK loss is negligible compared to other losses. Figure 1. Figure 1(a) assumes a constant BER, and Figure 1(b) assumes SNR to have a log-normal distribution. The shapes of the curves are noticeably different. This is because in the case where SNR is modeled probabilistically, the actual value of SNR has a much higher impact on an individual packet's successful transmission than the packet's length. While a constant BER model might suggest using a packet length of only 400 bytes, a more accurate model including SNR distribution shows that maximum packet length would be superior.

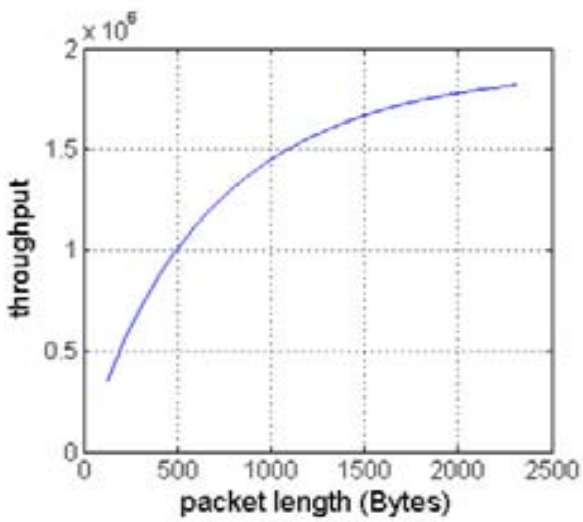

Figure 1: Throughput vs packet length assuming no collisions for (a) SNR fixed at 9dB, and (b) SNR with mean of 9dB with standard deviation of $3 \mathrm{~dB}$.

\section{B. Dynamic Packet Length Adaptation:}

Wireless link qualities can be greatly affected by environmental factors at different locations. To see how link diversity is, we setup one TelosB mote to measure the received signal strength at a resolution of $1 \mathrm{~ms}$. The experiments are conducted both indoor (the environment is noisy because of 802.11 interferences) and outdoor (the environment is quiet), respectively. We can see that the channel conditions vary drastically: in the noisy environment the RSSI value can be as high as $-62 \mathrm{dbm}$ while in the quiet environment the RSSI value can be as low as -96 $\mathrm{dbm}$. This indicates that packet length adaption schemes must be dynamically adapted to physical channel conditions to deliver performance gains in WSNs with spatial-temporal diversity in link qualities.

\section{Accuracy of Link Estimation:}

We evaluate the accuracy of the link estimation method in terms of absolute error. Each node transmits 200 packets in turn. All other nodes record each packet's reception. The estimated link qualities are compared against the ground truth values. Figure 8 shows the CDF of absolute error (i.e., estimated value - real value). We can see that the errors keep within $10 \%$ for $95 \%$ links, indicating that the link estimation method yields accurate results.

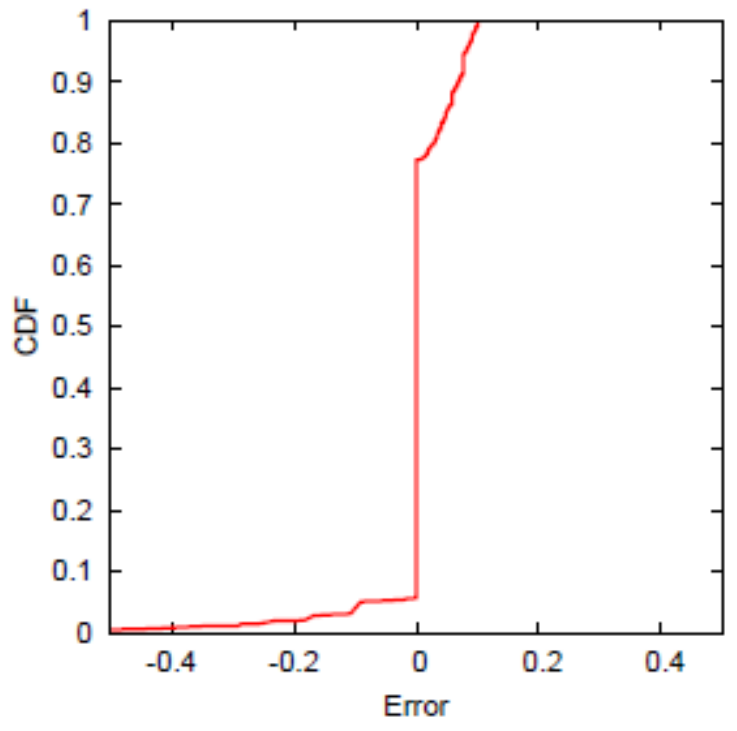

Figure 2: Accuracy of link estimation

\section{Comparative Analysis}




\section{International Journal of Science and Research (IJSR) \\ ISSN (Online): 2319-7064}

Index Copernicus Value (2013): 6.14 | Impact Factor (2014): 5.611

\begin{tabular}{|c|c|c|c|c|}
\hline Paper & $\begin{array}{c}\text { Packet } \\
\text { Loss }\end{array}$ & $\begin{array}{c}\text { Average } \\
\text { Delay }(\mathrm{ms})\end{array}$ & $\begin{array}{l}\text { Bit- Distortion-rate } \\
\text { performance for } \\
\text { streaming }(\mathrm{b} / \mathrm{s})\end{array}$ & Scheme /Algorithms \\
\hline $\begin{array}{c}\text { Packet Data } \\
\text { Transmission Over } \\
\text { Mobile } \\
\text { Radio Channels }\end{array}$ & $7.01 \%$ & 314 & 124 & $\begin{array}{c}\text { Network path } \\
\text { diversity, streaming of } \\
\text { packetized length. }\end{array}$ \\
\hline $\begin{array}{l}\text { Dynamic Packet } \\
\text { Length Control in } \\
\text { Wireless } \\
\text { Sensor Networks }\end{array}$ & $9.13 \%$ & 421 & 122 & $\begin{array}{l}\text { Error-cost optimized } \\
\text { transmission of a } \\
\text { single data } \\
\text { unit in isolation. }\end{array}$ \\
\hline $\begin{array}{c}\text { Packet Length } \\
\text { Adaptation in WLANs } \\
\text { with Hidden } \\
\text { Nodes and Time- } \\
\text { Varying Channels }\end{array}$ & $5.21 \%$ & 214 & 124 & $\begin{array}{l}\text { Multiple access } \\
\text { networks, integer } \\
\text { programming } \\
\text { problem. }\end{array}$ \\
\hline $\begin{array}{l}\text { Adaptive Design for } \\
\text { the Packet Length of } \\
\text { IEEE } \\
802.1 \text { n Networks }\end{array}$ & $2.12 \%$ & 131 & 54 & $\begin{array}{l}\text { Cross-layer } \\
\text { optimization, optimal } \\
\text { packet length, } \\
\text { distributed } \\
\text { coordination } \\
\text { function (DCF). }\end{array}$ \\
\hline
\end{tabular}

\section{Research Design}

In this section, we present the design of DPLC, a dynamic packet length control scheme for WSNs. Below, we identify the major design goals.

- Dynamic adaptation. DPLC should provide a dynamic adaptation scheme to achieve performance improvements in dynamic, time-varying sensor networks.

- Accurate link estimation. DPLC should implement an accurate link estimation method that can capture both physical channel conditions (due to channel fading, mobility, or power degradation) and interferences (from exposed and hidden terminals).

- Ease of programming. DPLC should provide easy-touse services to facilitate upper-layer application programming.

- Lightweight for implementation. DPLC should be lightweight for resource constrained sensor nodes.

\section{Flow Chart}

The flowchart of dynamic packet length optimization approach is described with the following diagram:

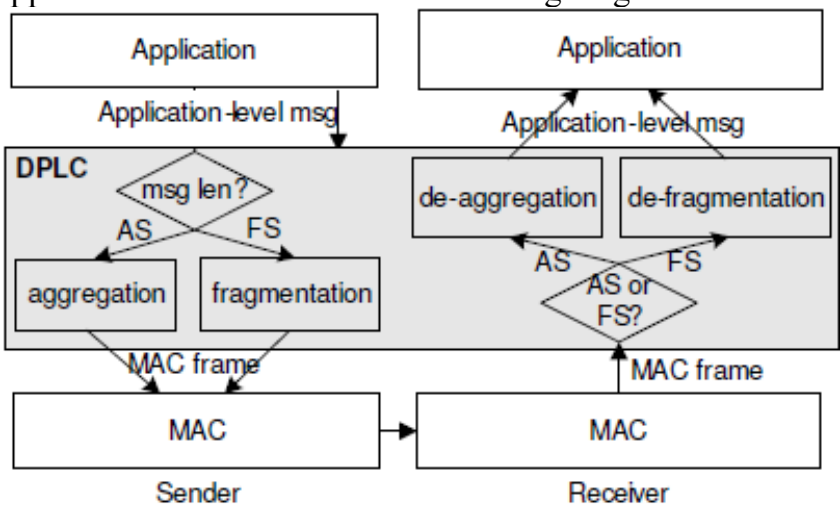

Figure 3: DPLC Overview

\section{Proposed Methodology}

The DPLC scheme works as follows. The application passes an application-level message for transmission. The DPLC module at the sender decides whether to use the aggregation service (AS, if the message length is small) or the fragmentation service (FS, if the message length is larger than the maximum packet length supported by the radio, i.e., 128 bytes for CC2420). The link estimator within DPLC dynamically estimates the appropriate packet length for transmission. Based on this, the DPLC module at the sender decides how many messages should be aggregated (for AS), or how many frames the message should be fragmented into (for FS). When a frame is ready for transmission (enough messages have been aggregated or time is out in AS), DPLC transmits it out via the MAC layer. When the DPLC module at the receiver receives a MAC frame, it deaggregates or defragments the frame in order to obtain the original message. When the message is ready (all frames in the message havebeen received or the receive buffer is full in FS), the DPLC module at the receiver notifies the upper layer for further handling. The DPLC scheme provides two services for upperlayer applications, i.e., the aggregation service (AS, for small messages) and the fragmentation service (FS, for large messages). (i) AS is useful for small data collection, e.g., CTP [7].

Both AS 8 and ASn requires L2 ACKs provided by the link layer, because packets need to be retransmitted (at least once) when they are lost. For AS 0, we additionally provide a more efficient ACK scheme called AggAck that does not rely on L2 ACKs, and thus mitigate the ACK overhead we use AS 0-L2 to denote AS 0 with L2 ACKs and AS0-AA to denote AS0 with AggAck afterwards). (ii) FS is useful for bulk data transmission, e.g., Flush. FS provides reliable transmissions as a large message is usually very important for upper-layer applications. FS does not necessarily depend on L2 ACKs. As mentioned above, we additionally provide the AggAck mechanism to mitigate the ACK overhead, and 


\section{International Journal of Science and Research (IJSR) \\ ISSN (Online): 2319-7064 \\ Index Copernicus Value (2013): 6.14 | Impact Factor (2014): 5.611}

more importantly, to deal with data packet retransmissions (we use FS-L2 to denote FS with L2 ACKs and FS-AA to denote FS with AggAck afterwards).

\section{Impact of Dynamic Optimization Mechanism}

This mechanism proposed here will provide the accurate link estimation, efficient utilization of channel in outdoor environment. This will improve the system throughput and packet delivery ratio in wireless sensor networks.

\section{Expected Outcome}

This dynamic packet length optimization appraoach provide the packet delivery ratio keeps high i.e. 95\% above and link estimated error within $10 \%$ for $95 \%$ link.

\section{Acknowledgement}

I am currently pursuing M.E in Computer Network and the work in this paper is under process. The dynamic packet length optimization approach will be implemented using the software modules. Further, dynamic packet length optimization approach will be used for accuracy of link estimation, efficient channel utilization and increase system throughput.

\section{Conclusion}

This proposed dynamic packet length optimization approach will provide accuracy in link estimation that capture physical channel condition, increase packet delivery ratio, increase system throughput and efficient energy utilization.

\section{References}

[1] Wei Dong, Chun Chen, Xue Liu, Yuan He,Yunhao Liu, Jiajun Bu, Xianghua $\mathrm{Xu}$, “ Dynamic Packet Length Control in Wireless Sensor Network," in Proc. 2014 IEEE Transactions.

[2] M. N. Krishnan, E. Haghani, and A. Zakhor, "Packet length adaptation in WLANs with hidden nodes and timevarying channels," in Proc. 2011 IEEE GlobeCom.

[3] W. Dong, X. Liu, C. Chen, Y. He, G. Chen, Y. Liu, and J. $\mathrm{Bu}$, "DPLC: dynamic packet length control in wireless sensor networks,"in Proc.2010 IEEE INFOCOM.

[4] B. Chen, Z. Zhou, Y. Zhao, and H. Yu, "Efficient error estimating coding: feasibility and applications," in Proc. 2010 ACM SIGCOMM.

[5] J. Huang, G. Xing, G. Zhou, and R. Zhou, "Beyond coexistence: exploiting WiFi white space for ZigBee performance assurance," in Proc. 2010 IEEE ICNP.

[6] O. Gnawali, R. Fonseca, K. Jamieson, D. Moss, and P. Levis, "Collection tree protocol," in Proc. 2009 ACM SenSys.

[7] Ganeriwal, I. Tsigkogiannis, H. Shim, V. Tsiatsis, M. B. Srivastava, and D. Ganesan, "Estimating clock uncertainty for efficient duty- cycling in sensor networks," IEEE/ACM Trans. Netw., vol. 17, no. 3, pp. 843-856, 2009.

[8] P. R. Jelenkovi'c and J. Tan, "Dynamic packet fragmentation for wireless channels with failures," in Proc. 2008 ACM MobiHoc.
[9] G. Hackmann, O. Chipara, and C. Lu, "Robust topology control for indoor wireless sensor networks," in Proc. 2008 ACM SenSys.

[10]F. Zheng and J. Nelson, "Adaptive design for the packet length of IEEE 802.11n networks," in Proc. 2008 IEEE ICC. 